\title{
INTEGRABILITY OF ROUGH ALMOST COMPLEX STRUCTURES
}

\author{
C. Denson Hill and Michael Taylor
}

\begin{abstract}
We extend the Newlander-Nirenberg theorem to manifolds with almost complex structures that have somewhat less than Lipschitz regularity. We also discuss the regularity of local holomorphic coordinates in the integrable case, with particular attention to Lipschitz almost complex structures.
\end{abstract}

\section{Introduction}

If $\Omega$ is a smooth manifold of dimension $2 n$, an almost complex structure on $\Omega$ is a section of $\operatorname{End}(T \Omega)$ such that $J^{2}=-I$. It is integrable provided there is a local coordinate chart about any $p \in \Omega$ consisting of holomorphic functions, where $f: \Omega \rightarrow \mathbb{C}$ is said to be holomorphic on $\mathcal{O} \subset \Omega$ provided

$$
(X+i J X) f=0
$$

on $\mathcal{O}$ for all smooth real vector fields $X$ on $\mathcal{O}$. The formal integrability condition is that the Lie bracket of complex vector fields of the form (1.1) continues to have such a form. Equivalently, one forms the Nijenhuis tensor:

$$
N(X, Y)=[X, Y]-[J X, J Y]+J[X, J Y]+J[J X, Y],
$$

which is linear over $C^{1}(\Omega)$ and hence defines a tensor field. The formal integrability condition is that $N$ vanish. The Newlander-Nirenberg theorem [NN] asserts that formal integrability implies integrability. In $[\mathrm{NN}]$ is was assumed that $J$ had a high degree of smoothness. Proofs in [NW] and [Mal] obtained the result for $J \in$ $C^{1+\varepsilon}, \varepsilon>0$. See also [Web]. Our goal here is to lower the needed regularity of $J$. We will establish the following.

Theorem 1.1. If $J$ is an almost complex structure on $\Omega$, satisfying

$$
J \in C^{r} \cap H^{s, p}, \quad r+s>1, \quad s p>2 n,
$$

with $0<r<s, 1<p<\infty$, then formal integrability implies integrability. 
Here $C^{r}$ is a Hölder space and $H^{s, p}$ is an $L^{p}$-Sobolev space. For convenience we will work under the hypothesis that also $s<1$. (But see some comments in $§ 5$.)

Our demonstration follows the method of [Mal], but it makes use of paraproduct analysis and some elliptic regularity results that were perhaps not familiar when the papers cited above were written. As a warm-up, we show that $N$ is well defined when $J$ satisfies (1.3). Since the components of $N$ involve products of components of $J$ and first-order derivatives of such components, it suffices to show the following.

Lemma 1.2. Assume $0<r<s<1$ and

$$
u, v \in C^{r} \cap H^{s, p}, \quad r+s>1,1<p<\infty .
$$

Then $u\left(\partial_{j} v\right)$ is a well defined distribution. Furthermore, if $u$ and $v$ are defined on a space of dimension $m$,

$$
s p \geq m \Longrightarrow u\left(\partial_{j} v\right) \in C_{*}^{r-1} \cap H^{s-1, p} .
$$

Proof. We use the paraproduct of J.-M. Bony [Bon] to write

$$
u\left(\partial_{j} v\right)=T_{u}\left(\partial_{j} v\right)+T_{\partial_{j}} u+R\left(u, \partial_{j} v\right)
$$

We have

$$
u \in L^{\infty} \Longrightarrow T_{u} \in O P \mathcal{O B} S_{1,1}^{0}
$$

the class of operators in $O P S_{1,1}^{0}$ defined in [Mey]. See also [T], Chapter 3, whose notation we follow here. Compare (1.6) with (3.5.1)-(3.5.2) of [T]. Now

$$
T_{u} \in O P \mathcal{O P} S_{1,1}^{0}, v \in C^{r} \cap H^{s, p} \Longrightarrow T_{u}\left(\partial_{j} v\right) \in C_{*}^{r-1} \cap H^{s-1, p}
$$

where $C_{*}^{s}$ denotes the scale of Zygmund spaces, defined for $s \in \mathbb{R}$ and coinciding with Hölder space $C^{s}$ for $s \in \mathbb{R}^{+} \backslash \mathbb{Z}^{+}$. Next, if $r \in(0,1)$,

$$
\begin{aligned}
v \in C^{r} & \Longrightarrow T_{\partial_{j} v} \in O P \mathcal{O P S} S_{1,1}^{1-r} \\
& \Longrightarrow T_{\partial_{j} v} u \in C_{*}^{2 r-1} \cap H^{r+s-1, p},
\end{aligned}
$$

when $u \in C^{r} \cap H^{s, p}$; cf. [T], (3.5.7). Finally,

$$
v \in C^{r} \Longrightarrow R_{\partial_{j} v} \in O P S_{1,1}^{1-r}
$$

where we set $R_{g} u=R(u, g)$ (cf. [T], (3.5.11)), and we have

$$
u \in H^{s, p} \Longrightarrow R_{\partial_{j} v} u \in H^{r+s-1, p}, \text { provided } r+s-1>0
$$

For (1.5), it suffices to have $H^{r+s-1, p} \subset C_{*}^{r-1}$, which holds if $s p \geq m$. This proves the lemma. 
1.2. A pseudodifferential operator $p(x, D)$ is said to belong to $O P S_{1, \delta}^{m}$ provided its symbol $p(x, \xi)$ satisfies

$$
\left|D_{x}^{\beta} D_{\xi}^{\alpha} p(x, \xi)\right| \leq C_{\alpha \beta}(1+|\xi|)^{m-|\alpha|+\delta|\beta|} .
$$

Operator results on $p(x, D)$ include

$$
p(x, D): H^{r+m, p} \rightarrow H^{r, p}, \quad p(x, D): C_{*}^{r+m} \rightarrow C_{*}^{r},
$$

assuming $1<p<\infty$. If $0 \leq \delta<1$, then (1.13) holds for all $r \in \mathbb{R}$. If $\delta=1$, one needs $r>0$ for (1.13) to hold. One says $p(x, D) \in O P \mathcal{B} S_{1,1}^{m}$ provided (1.12) holds with $\delta=1$ and also the partial Fourier transform $\hat{p}(\eta, \xi)$ satisfies

$$
\operatorname{supp} \hat{p}(\eta, \xi) \subset\{(\eta, \xi):|\eta| \leq a|\xi|\},
$$

for some $a<1$. For such operators, (1.13) holds for all $r$.

REMARK 1.2. Another consequence of the operator results mentioned in Remark 1.1 is that

$$
F \in C^{\infty}, \quad u \in C_{*}^{r} \cap H^{s, p} \Longrightarrow F(u) \in C_{*}^{r} \cap H^{s, p},
$$

given $r, s>0, p \in(1, \infty)$. In fact, tools developed in [Bon] yield

$$
F(u)=F(0)+M(x, D) u, \quad u \in L^{\infty} \Rightarrow M(x, D) \in O P S_{1,1}^{0},
$$

and then (1.15) follows from (1.13). One can have $u=\left(u_{1}, \ldots, u_{K}\right)$, so in particular $C_{*}^{r} \cap H^{s, p}$ contains $u v$ if it contains $u$ and $v$, and it also contains $u^{-1}$ if this inverse is continuous.

REMARK 1.3. Our notation above does not specify domains on which elements of $C^{r}, H^{s, p}$, etc., are to be defined. For example, we could take $C_{*}^{r}\left(\mathbb{R}^{m}\right), H^{r, p}\left(\mathbb{R}^{m}\right)$, etc., in (1.13). Of course a nonzero constant (perhaps $F(0)$ ) does not belong to $H^{s, p}\left(\mathbb{R}^{m}\right)$, but in this context we intend to use $H^{s, p}(U)$ and $C^{r}(\bar{U})$ for some bounded $U \subset \mathbb{R}^{m}$. The result (1.15) holds in this context. To justify it one extends $u$ to an element of $C_{*}^{r} \cap H^{s, p}$ on a neighborhood of $\bar{U}$ and makes use of (1.13) in such a situation, as is standard. Lemma 1.2 also holds for $u, v \in C^{r}(\bar{U}) \cap H^{s, p}(U)$, and this will be useful in $\S 3$.

REMARK 1.4. Another consequence of Lemma 1.2, together with Remark 1.2, is that the quantity (1.2) is well defined for all vector fields $X$ and $Y$ that are regular of class $C^{r} \cap H^{s, p}$, under the hypothesis (1.3), and if $N(X, Y)$ vanishes for all smooth vector fields then it vanishes for all such vector fields. This class of vector fields is invariant under diffeomorphisms of class $C^{1+r} \cap H^{1+s, p}$, and this fact will be of use in $\S 3$.

REMARK 1.5. We dwell a little on the conditions under which the regularity hypothesis (1.3) holds. Note that one special case is 
Now if

$$
J \in C^{r}, \quad r>\frac{1}{2}
$$

then $J \in H^{1 / 2+\varepsilon, p}$ for all $p<\infty$ as long as $1 / 2+\varepsilon<r$, so Theorem 1.1 applies whenever (1.18) holds.

The following outlines the rest of this paper. In $\S 2$ we rephrase the problem as an overdetermined system of PDEs on an open set in $\mathbb{C}^{n}$, and give an integrability condition equivalent to $N \equiv 0$. In $\S 3$ we discuss Malgrange's factorization technique, which is to write the local coordinate chart $F=\left(f_{1}, \ldots, f_{n}\right)$ as $G \circ H$; in this section we construct $H$. In $\S 4$ we show that the construction of $G$ is a consequence of the classical real analytic theory. All this is in direct parallel to [Mal], and the new material in these sections consists of demonstrations that the various steps work under our weakened regularity hypotheses. In $\S 5$ we make some concluding comments about the degree of regularity of the holomorphic coordinates when hypothesis (1.3) holds, and we also make special note of the situation when $J$ is Lipschitz. In such a case, we show the components of $F$ have two derivatives in bmo.

\section{Preliminaries}

As in [Mal], we identify a neighborhood of $p$ in $\Omega$ with a neighborhood of the origin 0 in $\mathbb{C}^{n}$ and arrange that $J(0)$ coincide with the standard complex structure on $\mathbb{C}^{n}$. The task of solving (1.1) on a neighborhood of $p$ for a family $f_{1}, \ldots, f_{n}: \mathcal{O} \rightarrow$ $\mathbb{C}$ forming a local coordinate system becomes that of solving an overdetermined system of the form

$$
\frac{\partial f_{\ell}}{\partial \bar{z}_{j}}=\sum_{k} a_{j k} \frac{\partial f_{\ell}}{\partial z_{k}}, \quad 1 \leq j, \ell \leq n .
$$

The hypothesis (1.3) is equivalent to $a_{j k} \in C^{r} \cap H^{s, p}$, with $r, s, p$ as in the statement of Theorem 1.1. It will be convenient to allow a little wriggle room, when we work with (3.9), so we will actually assume

$$
a_{j k} \in C^{r_{1}} \cap H^{s, p}, \quad r_{1}>r, \quad a_{j k}(0)=0 .
$$

This does not affect the validity of Theorem 1.1 as stated. The condition $a_{j k}(0)=0$ just reflects our normalization of $J(0)$.

In (2.1), $z_{j}=x_{j}+i y_{j}$ form the standard coordinates on $\mathbb{C}^{n}$ and as usual we set $\partial / \partial \bar{z}_{j}=(1 / 2)\left(\partial / \partial x_{j}+i \partial / \partial y_{j}\right)$, etc. A convenient shorthand is to set $\partial / \partial \bar{z}=$ $\left(\partial / \partial \bar{z}_{1}, \ldots, \partial / \partial \bar{z}_{n}\right)^{t}$ (a column vector), $A_{j}=\left(a_{j 1}, \ldots, a_{j n}\right)$ (a row vector), $A=$ $\left(a_{j k}\right)$, and $F=\left(f_{1}, \ldots, f_{n}\right)$ (a row vector). Then $(2.1)$ is written 
The integrability condition $N \equiv 0$ becomes

$$
\frac{\partial A_{j}}{\partial \bar{z}_{k}}+A_{j} \frac{\partial A_{k}}{\partial z}=\frac{\partial A_{k}}{\partial \bar{z}_{j}}+A_{k} \frac{\partial A_{j}}{\partial z}, \quad 1 \leq j, k \leq n .
$$

Note that Lemma 1.2 applies directly to (2.4). The goal will be to construct a solution $F \in C^{1+r} \cap H^{1+s, p}$ to $(2.3)$, with $F$ close to the identity map in $C^{1}$-norm, under the assumption that (2.4) holds. This will provide the desired holomorphic coordinates.

\section{Malgrange factorization}

A key idea in [Mal] was to produce $F$ in the form

$$
F=G \circ H,
$$

and to apply separate techniques to construct the diffeomorphisms $G$ and $H$. These factors will be arranged to satisfy

$$
\frac{\partial G}{\partial \bar{\zeta}}=B \frac{\partial G}{\partial \zeta}
$$

with $\zeta=H(z)$, and

$$
\frac{\partial H}{\partial \bar{z}}+\frac{\partial \bar{H}}{\partial \bar{z}}(B \circ H)=A\left[\frac{\partial H}{\partial z}+\frac{\partial \bar{H}}{\partial z}(B \circ H)\right]
$$

and furthermore it is arranged that

$$
\sum_{j} \frac{\partial B_{j}}{\partial \zeta_{j}}=0
$$

Here we need to verify that the construction works under the regularity hypothesis (2.2). First, as computed in [Mal], if (3.1) holds with $G, H \in C^{1}$ and if (3.2) holds, then (2.3) is equivalent to (3.3). For the next lemma we take $r, s, p$ as in the statement of Theorem 1.1, and we assume (2.2) holds.

Lemma 3.1. Assume $H \in C^{1+r} \cap H^{1+s, p}$ and that $H$ is sufficiently close to the identity in $C^{1}$-norm, and use (3.3) to define $B \circ H \in C^{r} \cap H^{s, p}$, i.e.,

$$
B \circ H=-\left(\frac{\partial \bar{H}}{\partial \bar{z}}-A \frac{\partial \bar{H}}{\partial z}\right)^{-1}\left(\frac{\partial H}{\partial \bar{z}}-A \frac{\partial H}{\partial z}\right) .
$$

Then also $B \in C^{r} \cap H^{s, p}$. If $A$ verifies the formal integrability condition (2.4), then so does $B$, i.e.,

$$
\frac{\partial B_{j}}{\partial \bar{\zeta}_{k}}+B_{j} \frac{\partial B_{k}}{\partial \zeta}=\frac{\partial B_{k}}{\partial \bar{\zeta}_{j}}+B_{k} \frac{\partial B_{j}}{\partial \zeta}, \quad 1 \leq j, k \leq n
$$

Proof. As (2.4) restates the formal integrability of $J$, i.e., the vanishing of (1.2) for all vector fields $X, Y \in C^{r} \cap H^{s, p}$, the system (3.6) restates the formal integrability of $\widetilde{J}$, given by

$$
\widetilde{J}(\zeta)=D H(z) J(z) D H(z)^{-1}, \quad \zeta=H(z) .
$$

Note that under the stated regularity hypotheses on $J$ and $H$ we have $\widetilde{J} \in C^{r} \cap H^{s, p}$. Now the equivalence of the formal integrability of $J$ and $\widetilde{J}$ just amounts to the coordinate invariance of (1.2). 
Lemma 3.2. Given $\varepsilon, \delta>0$, one can find a ball $U$ about $0 \in \mathbb{C}^{n}$ and

$$
H \in C^{1+r}(\bar{U}) \cap H^{1+s, p}(U)
$$

satisfying

$$
H(0)=0, \quad\|H-i d\|_{C^{1+r}(\bar{U})}<\delta
$$

and such that $B \in C^{r}(\bar{U}) \cap H^{s, p}(U)$, defined by (3.5), satisfies $|B(0)|<\varepsilon$ and also satisfies (3.4).

In our proof, following [Mal], we find it convenient to fix $U$ and dilate $A$, setting $A_{t}(z)=A(t z)$. Let $E=B \circ H$, so

$$
E=-\left(\frac{\partial \bar{H}}{\partial \bar{z}}-A_{t} \frac{\partial \bar{H}}{\partial z}\right)^{-1}\left(\frac{\partial H}{\partial \bar{z}}-A_{t} \frac{\partial H}{\partial z}\right)
$$

The map $\Phi(H, t)=E$ has the mapping property

$$
\Phi: \mathcal{B}^{r, s, p}(\delta) \times[0,1] \longrightarrow C^{r}(\bar{U}) \cap H^{s, p}(U)
$$

where $\mathcal{B}^{r, s, p}(\delta)$ consists of $H \in C^{1+r}(\bar{U}) \cap H^{1+s, p}(U)$ satisfying (3.8). Then both $\Phi$ and $D_{H} \Phi$ are continuous, where we regard our Banach spaces as real Banach spaces. An application of the chain rule gives

$$
\frac{\partial B_{j}}{\partial \zeta_{j}} \circ H=\left(\frac{\partial K}{\partial \zeta_{j}} \circ H\right) \frac{\partial E_{j}}{\partial z}+\left(\frac{\partial \bar{K}}{\partial \zeta_{j}} \circ H\right) \frac{\partial E_{j}}{\partial \bar{z}}, \quad K=H^{-1}
$$

One can express $\left(\partial K / \partial \zeta_{j}\right) \circ H$ and $\left(\partial \bar{K} / \partial \zeta_{j}\right) \circ H$ in terms of the $z$ - and $\bar{z}$-derivatives of $H$ and $\bar{H}$, using the identity

$$
(D K) \circ H(x)=D H(x)^{-1}
$$

of $(2 n) \times(2 n)$ real matrices. It follows via Lemma 1.2 (extended to function spaces on bounded domains) that

$$
\Psi(H, t)=\sum_{j} \frac{\partial B_{j}}{\partial \zeta_{j}} \circ H
$$

defines a map

$$
\Psi: \mathcal{B}^{r, s, p}(\delta) \times[0,1] \longrightarrow C_{*}^{r-1}(\bar{U}) \cap H^{s-1, p}(U)
$$

which is continuous, along with $D_{H} \Psi$. Note that $\Psi(i d, 0)=0$. A calculation gives

$$
D_{H} \Psi(i d, 0) h=\sum_{j} \frac{\partial^{2} h}{\partial z_{j} \partial \bar{z}_{j}}=\frac{1}{4} \Delta h .
$$

This map has a right inverse 
where $\mathcal{G}$ denotes the solution operator to

$$
\Delta v=h \text { on } U,\left.\quad v\right|_{\partial U}=0
$$

This has the crucial mapping properties

$$
\mathcal{G}: C_{*}^{r-1}(\bar{U}) \rightarrow C^{r+1}(\bar{U}), \quad \mathcal{G}: H^{s-1, p}(U) \rightarrow H^{s+1, p}(U)
$$

valid for $0<r, s<1,1<p<\infty$. These properties of $\mathcal{G}$ can be established by extending $h$ to a neighborhood of $\bar{U}$, using local regularity, and then using well known regularity of harmonic functions on $\bar{U}$ with boundary values in $C^{r+1}(\partial U)$ or $B_{p, p}^{s+1-1 / p}(\partial U)$, respectively. The implicit function theorem yields for small $t>0$ a solution to $\Psi(H, t)=0$ close to the solution $H_{0}(z)=z$ to $\Psi\left(H_{0}, 0\right)=0$. This proves the lemma.

REMARK 3.1. It is for the continuity in $t$ in (3.10) and (3.13) that we need $r_{1}>r$ in (2.2). Also for this reason we need $s p>2 n$ in (1.3), rather than the weaker inequality that suffices for (1.5).

\section{Reduction to the analytic case}

At this point it remains to construct a diffeomorphism $G \in C^{1+r}$ satisfying (3.2), where $B \in C^{r} \cap H^{s, p}$ satisfies (3.4) and (3.6). The key observation of [Mal] is that this forces $B$ to be real analytic. Our final task is to verify that this works under our weaker regularity hypothesis.

Lemma 4.1. If $r, s, p$ satisfy the hypotheses of Theorem 1.1 and (3.4) and (3.6) hold for $B \in C^{r} \cap H^{s, p}$, then $B$ is real analytic, on a neighborhood of $0 \in \mathbb{C}^{n}$.

Proof. Since $|B(0)|<\varepsilon$, the system (3.4), (3.6) is an overdetermined elliptic system for $B$ on a neighborhood of the origin. Once we show $B \in C^{\infty}$, the real analyticity is classical. The smoothness of $B$ follows from Theorem 2.2.E of [T], but for the reader's convenience we sketch a proof.

The overdetermined elliptic system (3.4), (3.6) has the form

$$
L B+K(B, \nabla B)=0
$$

where $L$ is a first-order linear operator (with constant coefficients, in this case) and $K(B, \nabla B)$ is bilinear in its arguments. Ellipticity near $z=0$ follows from $|B(0)|<\varepsilon$. Using the sort of symbol smoothing discussed in Chapter 3 of [T], we can write

$$
K(B, \nabla B)=M^{\#}(x, D) B+M^{b}(x, D) B
$$

and, by (3.3.25) of [T], given $r>0$, 
Here $\delta \in(0,1)$ is picked in advance. Also we have $L+M^{\#}(x, D) \in O P S_{1, \delta}^{1}$ elliptic, with left parametrix $E \in O P S_{1, \delta}^{-1}$. Hence, if $\delta$ is close enough to 1, we have

$$
B=-E M^{b}(x, D) B, \quad \bmod C^{\infty}
$$

and

$$
\begin{aligned}
B \in C^{r} \cap H^{s, p}, r+s>1 & \Longrightarrow M^{b}(x, D) B \in H^{s+r \delta-1, p} \\
& \Longrightarrow B \in H^{s+r \delta, p} .
\end{aligned}
$$

We can iterate (4.4)-(4.5) arbitrarily often to obtain $B \in C^{\infty}$.

Having Lemma 4.1, the endgame is that given in [Mal]. Since $B$ is real analytic, the Cartan-Kahler theorem implies (3.2) is solvable, for a real analytic diffeomorphism $G$, given the integrability condition (3.6).

\section{Further regularity results}

Here we make note of the regularity of the map $F$ in terms of the hypothesized regularity of the almost complex structure $J$. Here is one result.

Proposition 5.1. Under the hypothesis (1.3) on $J$, when $N \equiv 0$, then

$$
F \in C_{*}^{r+1} \cap H^{s+1, p}
$$

Once we show that $H$ has this regularity, then (5.1) will follow for $F=G \circ H$, as in Remark 1.2. This degree of regularity for $H$ was indicated in Lemma 3.2, but to establish this lemma we raised the regularity assumed on $J$, in (2.2), so, shifting back, at this point we merely have $H \in C_{*}^{\rho+1} \cap H^{s+1, p}$, for $r-\rho=\varepsilon>0$, arbitrarily small. We will be able to go from here to (5.1) via some elliptic regularity. In fact, the formulas (3.9) and (3.11) show that the condition (3.4) yields for $H$ an elliptic system of the form

$$
\sum_{j} a_{j}(\nabla H) \partial_{j} b_{j}(\nabla H, A \nabla H)=0
$$

with $a_{j}$ and $b_{j}$ smooth in its arguments and

$$
A \in C^{r} \cap H^{s, p}, \quad H \in C^{\rho+1} \cap H^{s+1, p},
$$

with $r-\rho=\varepsilon>0$, arbitrarily small. We continue to impose the conditions in (1.3) on $r, s, p$, and for simplicity we continue to assume $0<r<s<1$. The deduction that $H \in C^{r+1}$ is not a standard elliptic regularity result, but we can bring paraproduct techniques to bear to prove it.

To be sure, passing from $F \in C^{r+1-\varepsilon}$ to $F \in C^{r+1}$ is a small thing. In fact, the 
Proposition 5.2. If $J$ is Lipschitz and $N \equiv 0$, then

$$
\partial^{2} F \in \text { bmo . }
$$

Here bmo denotes the localized John-Nirenberg space. What we know from the results of $\S \S 3-4$ is that, under this hypothesis, $H \in C^{1+r}$ and hence $F \in C^{1+r}$, for all $r<1$. Again it remains to establish that $\partial^{2} H \in$ bmo when $H$ satisfies an elliptic system of the form (5.2) and we know that

$$
A \in \operatorname{Lip}, \quad H \in C^{1+\rho}, \quad \forall \rho<1
$$

and (equivalently) $H \in H^{s+1, p}, \forall s<1, p<\infty$.

To begin the proof, the paradifferential calculus described in Remark 1.2 gives

$$
b_{j}(\nabla H, A \nabla H)=B_{j 1} \nabla H+B_{j 2} A \nabla H \bmod C^{\infty},
$$

where we have $B_{j \nu} \in O P S_{1,1}^{0}$, and furthermore, upon choosing $\delta \in(0,1)$, we can write

$$
\begin{gathered}
\nabla H, A \nabla H \in C^{\rho} \Longrightarrow B_{j \nu}=B_{j \nu}^{\#}+B_{j \nu}^{b} \\
B_{j \nu}^{\#} \in O P S_{1, \delta}^{0}, \quad B_{j \nu}^{b} \in O P S_{1,1}^{-\rho \delta}
\end{gathered}
$$

Cf. [T], Proposition 3.1.D. Hence we have

$$
B_{j 1}^{b} \nabla H, B_{j 2}^{b} A \nabla H \in C_{*}^{\rho+\rho \delta} \cap H^{s+\rho \delta, p} .
$$

Thus (5.2) yields

$$
\sum_{j} a_{j}(\nabla H) \partial_{j}\left(B_{j 1}^{\#} \nabla H+B_{j 2}^{\#} A \nabla H\right)=f_{1}
$$

with

$$
f_{1}=-\sum a_{j}(\nabla H) \partial_{j}\left(B_{j 1}^{b} \nabla H+B_{j 2}^{b} A \nabla H\right) \in C_{*}^{\rho+\gamma-1} \cap H^{s+\gamma-1, p},
$$

for some $\gamma>0$, whose specific formula we do not need. Next, we analyze the product in (5.9) in terms of paraproducts, as in (1.6), obtaining

$$
\sum_{j} T_{a_{j}(\nabla H)} \partial_{j}\left(B_{j 1}^{\#} \nabla H+B_{j 2}^{\#} A \nabla H\right)=f_{2},
$$

with

$$
\begin{aligned}
& f_{2}=f_{1}-\sum T_{\psi_{j}} a_{j}(\nabla H)-\sum R\left(a_{j}(\nabla H), \psi_{j}\right), \\
& \psi_{j}=\partial_{j}\left(B_{j 1}^{\#} \nabla H+B_{j 2}^{\#} A \nabla H\right) .
\end{aligned}
$$

We have $\psi_{j} \in C_{*}^{\rho-1} \cap H^{s-1, p}$. Then, via (1.9), 
while, as in (1.11), given that $\rho$ is so close to $r$ that $\rho+s>1$,

$$
R\left(a_{j}(\nabla H), \psi_{j}\right) \in H^{\rho+s-1, p} \subset C_{*}^{\rho+\gamma-1}, \text { if } s p>2 n,
$$

where again $\gamma$ is a positive number that we need not compute. In summary,

$$
f_{2} \in C_{*}^{\rho+\gamma-1} .
$$

Next, we have

$$
T_{a_{j}(\nabla H)}=P_{j}^{\#}+P_{j}^{b}, \quad P_{j}^{\#} \in O P S_{1, \delta}^{0}, \quad P_{j}^{b} \in O P \mathcal{B} S_{1,1}^{-\rho \delta} .
$$

Then (5.11) yields

$$
\sum_{j} P_{j}^{\#} \partial_{j}\left(B_{j 1}^{\#} \nabla H+B_{j 2}^{\#} A \nabla H\right)=f_{3}
$$

where, with $\psi_{j}$ as in (5.12),

$$
f_{3}=f_{2}-\sum P_{j}^{b} \psi_{j} \in C_{*}^{\rho+\gamma-1}
$$

and again $\gamma>0$.

For the next step, we have

$$
A \nabla H=T_{A} \nabla H+T_{\nabla H} A+R(A, \nabla H)=T_{A} \nabla H+g .
$$

This is where the regularity of $A$ crucially affects the regularity of $H$. Given $\nabla H \in$ $C^{\rho}, 0<\rho<1$, we have, for $r>0$,

$$
A \in C_{*}^{r} \Rightarrow g \in C_{*}^{r}, \quad A \in \operatorname{Lip} \Rightarrow \nabla g \in \text { bmo } .
$$

Now (5.17) yields

$$
\sum_{j} P_{j}^{\#} \partial_{j}\left(B_{j 1}^{\#} \nabla H+B_{j 2}^{\#} T_{A} \nabla H\right)=f_{4}
$$

where, with $g$ as in (5.18)-(5.19),

$$
f_{4}=f_{3}-\sum P_{j}^{\#} \partial_{j} B_{j 2}^{\#} g
$$

Hence, given $0<\rho<r, \rho+\gamma>r$ (which can be assumed in this context),

$$
A \in C_{*}^{r} \Rightarrow f_{4} \in C_{*}^{r-1}, \quad A \in \operatorname{Lip} \Rightarrow f_{4} \in \text { bmo. }
$$

Furthermore, we have

$$
T_{A}=Q^{\#}+Q^{b}, \quad Q^{\#} \in O P S_{1, \delta}^{0}, \quad Q^{b} \in O P \mathcal{B} S_{1,1}^{-r \delta},
$$

where we can take $r=1$ if $A \in \operatorname{Lip}$. Then (5.20) yields

$$
\sum P_{j}^{\#} \partial_{j}\left(B_{j 1}^{\#} \nabla H+B_{j 2}^{\#} Q^{\#} \nabla H\right)=f_{5},
$$


with

$$
f_{5}=f_{4}-\sum P_{j}^{\#} \partial_{j} B_{j 2}^{\#} Q^{b} \nabla H
$$

which has as much regularity as $f_{4}$. Now the left side of (5.24) is of the form $P H$, where $P \in O P S_{1, \delta}^{2}$ is elliptic, and hence has a parametrix $E \in O P S_{1, \delta}^{-2}$. Hence

$$
H=E f_{5}, \bmod C^{\infty},
$$

and, by (5.22) and the analogue for $f_{5}$, given $0<r<1$,

$$
A \in C^{r} \Rightarrow H \in C^{r+1}, \quad A \in \operatorname{Lip} \Rightarrow \partial^{2} H \in \text { bmo . }
$$

This completes the proof of Propositions 5.1 and 5.2.

\section{References}

[Bon] J.-M. Bony, Calcul symbolique et propagation des singularitiés pour des équations aux dérivées nonlinéaires, Ann. Sci. Ecole Norm. Sup. 14 (1981), 209-246.

[Mal] B. Malgrange, Sur l'intégrabilité des structures presque-complexes, Symposia Math., Vol. II (INDAM, Rome, 1968), Academic Press, London, 1969, pp. 289-296.

[Mey] Y. Meyer, Remarques sur un théorème de J.M. Bony, Rend. del Circolo mat. di Palermo (suppl. II:1) (1981), 1-20.

[NN] A. Newlander and L. Nirenberg, Complex coordinates in almost complex manifolds, Ann. of Math. 65 (1957), 391-404.

[NW] A. Nijenhuis and W.B. Woolf, Some integration problems in almost-complex manifolds, Ann. of Math. 77 (1963), 424-489.

[T] M. Taylor, Pseudodifferential Operators and Nonlinear PDE, Birkhauser, Boston, 1991.

[Web] S. Webster, A new proof of the Newlander-Nirenberg theorem, Math. Zeit. 201 (1989), 303-316.

Math. Dept., State University of New York, Stony Brook NY 11794

Math. Dept., University of North Carolina, Chapel Hill NC 27599 